International Journal of Distributed and Parallel Systems (IJDPS) Vol.3, No.5, September 2012

\title{
Role of Multi Agent System For QoS GUARANTEE IN CELLULAR NETWORKS
}

\author{
Nupur Giri ${ }^{1}$ and Shrikant Bodhe ${ }^{2}$ \\ ${ }^{1}$ VESIT, Computer Engineering Department., Mumbai, India \\ nupur.giri@gmail.com \\ ${ }^{2}$ Principal, SVERI's College of Engineering, Pandharpur, Maharashtra, India. \\ s_k_bodhe@yahoo.com
}

\begin{abstract}
The paper highlights the benefits of Multi Agent System for maintaining QoS in cellular network by evaluating various multi agent based call admission control strategies for different traffic conditions. It establishes the effect of degree of distribution of agents on system performance by comparing the two service architectures, namely centralized and distributed, for reactivity, responsiveness, utilization of resources, communication overhead, sustainability, scalability, robustness and modifiability. It also establishes the relationship between the social attitude of an agent towards the other agents and fairness of resource distribution in distributed architecture. This evaluation helps in building knowledge for choosing the optimal multi agent based call admission and channel borrowing schemes, along with the most suitable service architecture for the required QoS and traffic conditions.
\end{abstract}

\section{KEYWORDS}

Multi Agent System, Service Architecture, Cellular Networks, Call Admission Control

\section{INTRODUCTION}

National Consumer Helpline, a project supported by the ministry of consumer affairs (India), estimates that as many as $20 \%$ of all calls made are dropped. Telecom Regulatory Authority of India (TRAI) has brought down the benchmark of call drop rate from $3 \%$ to $2 \%$ of the total number of successful calls. To make sure that the mobile service providers adhere to these regulations, the quality of service has to be strictly monitored.

Call Admission Control (CAC) [1,2] limits the number of call connections into the network in order to guarantee connection level Quality of Service (QoS) parameters. Velocity, direction, distance of travel and class of the call (voice or data) made by a mobile user also affects call admission decision for that call. While admitting calls to a cell, if information about ongoing calls in the neighboring cells is available, then the cell can derive the impact of neighboring calls, on its own call admission and perform resource allocation for handoff calls in advance. This reduces the handoff call dropping probability. Also in case of congestion or QoS degradation, channels are borrowed from the neighboring cells depending on their ongoing call status. This exchange of traffic information requires neighboring cells to interact with each other in cooperative manner and can be modeled as a Multi Agent System $[3,4]$.

A Multi Agent System (MAS) comprises of a set of agents, which interact, collaborate, cooperate, or even negotiate with each other and with the environment to solve a particular problem in a coordinated manner.

The work presented in the paper contributes by demonstrating the ability of MAS in improving or maintaining the quality of service as required by cellular network provider. This is achieved 
through agent interaction, for various call admission control strategies for different traffic classes.

\section{REVIEW STAGE}

The first work on using intelligent hybrid agents to control mobile networks was demonstrated by Bodanese [5] which was about resource allocation scheme for first generation mobile networks that offered an efficient solution for resource allocation under moderate and heavy loads. More recent work was of European Union ACTS Projects [6, 7], IMPACT and FACTS, that portrayed hybrid agents as significant entities in managing resources between SPs and NPs. The European Commission's Information Society Technologies - 'Shuffle' project [8] proposed agents to control and manage both business interactions and radio resources. It presented hybrid agent based layered architecture for network management. The work offered business models of network providers, service providers and customers and the relationships between these actors.

Our work uses this business model, and is in the direction of performance evaluation of multi agent based CAC schemes, multi agent based service architecture and multi agent based dynamic channel borrowing schemes. It also evaluates how the degree of distribution of agents, manner of their interaction and their social attitude towards each other affects the performance parameters of the system.

\section{THE WORK}

A particular novel aspect of our work is the introduction of a new plane called 'Connection Plane' and the design of new cell level hybrid agent called 'Network Provider Cell Agent (NPCA)' as extension to the 'Shuffle' model as shown in figure 1. This agent is a layered hybrid agent, with 'Local Planning' and 'Call Establishment' layers as presented in figure 2. It has the capability of interacting with other agents in distributed manner (figure 3). This NPCA agent makes call admission and channel borrowing decision according to the policies passed by the Local Planning Layer. Designing the interaction model for agents and evaluation of performance for different CAC schemes forms the first part of our contributions.

Our work proposes two MAS based Service Architectures depending on the degree of distribution of agents and the type of interaction, in Connection Plane: 
International Journal of Distributed and Parallel Systems (IJDPS) Vol.3, No.5, September 2012

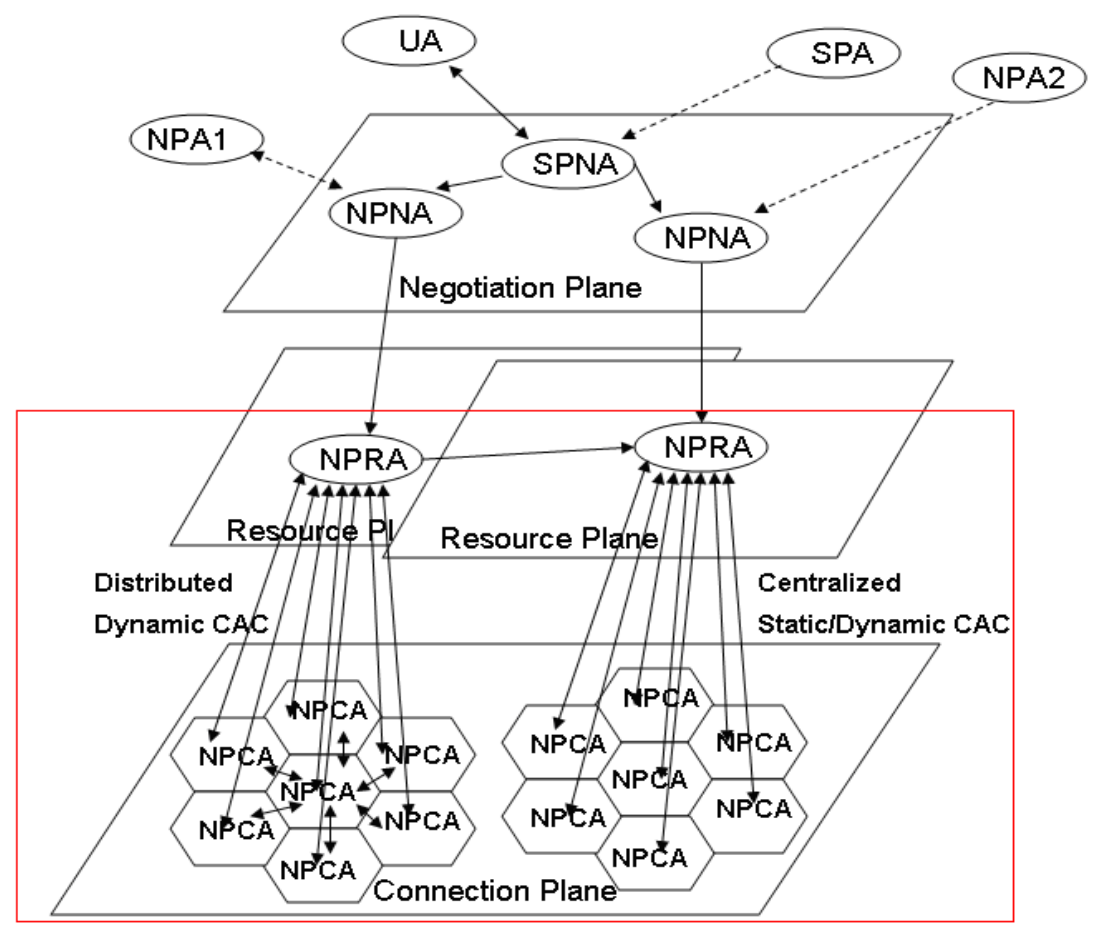

Figure1 . Functional Diagram: Extended 'Shuffle' Model

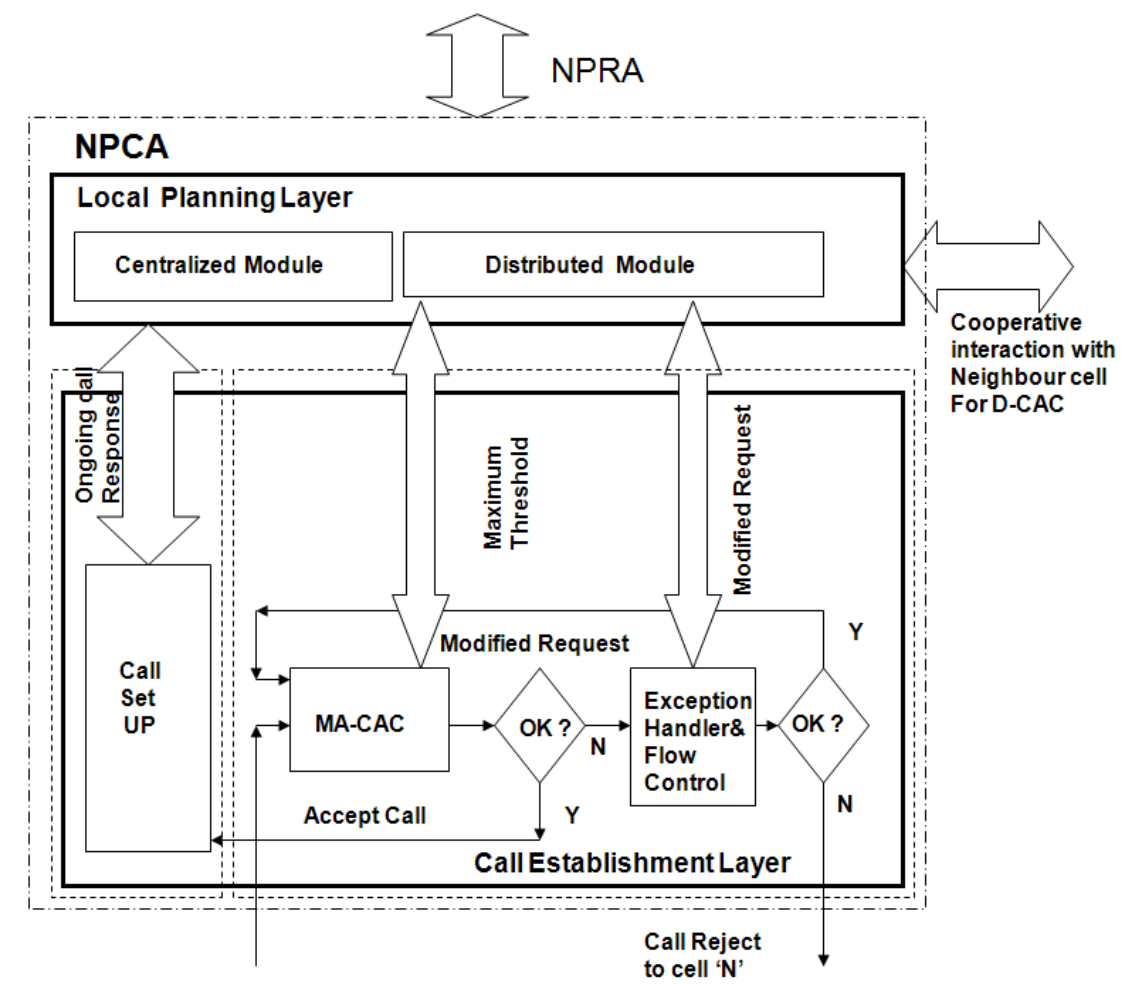

Figure 2. NPCA for Distributed Service Architecture -MAS 
Network Provider Resource Agent (NPRA-based) Centralized Service Architecture and Network Provider Cell Agent (NPCA-based) Distributed Service Architecture. These service architectures use Foundation for Intelligent Physical Agent compliant message 'performatives'[9]. These performatives have been modeled using open source Java Agent DEvelopment framework, JADE 3.1[10]. The comparisons of these two service architectures for reactivity, responsiveness, utilization of resources, communication overhead, sustainability, scalability, robustness and modifiability form the second part of our contribution.

The third part of our work deals with call admission control policies of Local Planning Layer of NPCA. These policies are termed as Multi Agent based CACs (MA-CAC). The admission policies chosen are static as well as dynamic. They also cater to different classes (voice/data) of traffic and mobility patterns (high/low). Priority and non-priority handoff traffic is also considered. Various MA-CACs were compared and performance evaluation of each in terms of connection level parameters such as new call blocking probability, handoff call blocking probability, effect of queue on blocking probability in multi agent based environment is presented.

The final part of our work uses the concept of Social Welfare for channel borrowing through MAS of socially intelligent agents to handle congestion.

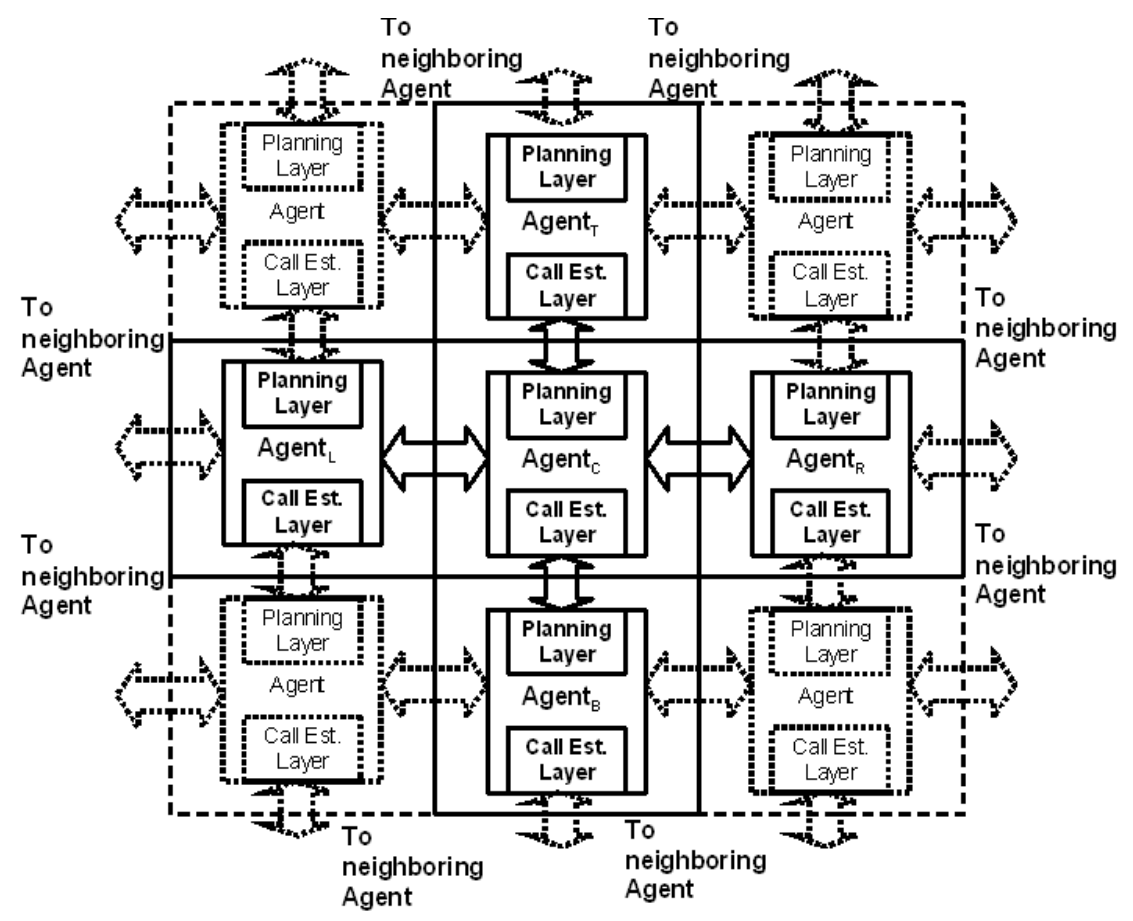

Figure 3. Distributed Service Architecture: NPCA Cluster Interaction

\subsection{Multi Agent Call Admission Control (MA-CAC)}

The MA-CAC module implemented Static Cutoff Priority (S-CAC)[11], Dynamic Cutoff Priority (D-CAC) [12] and Mobility Based Channel Reservation (MBCR) CAC schemes[13]. The S-CAC scheme admitted calls according to static cutoff threshold of ongoing calls, otherwise it is blocked. 
But this scheme was found to be unsuitable for real time traffic as the cut off thresholds were static and new call blocking and handoff call blocking probabilities were too high (figure 4,5). It did not meet the requirement of low handoff call blocking probability and call admission of real time traffic.

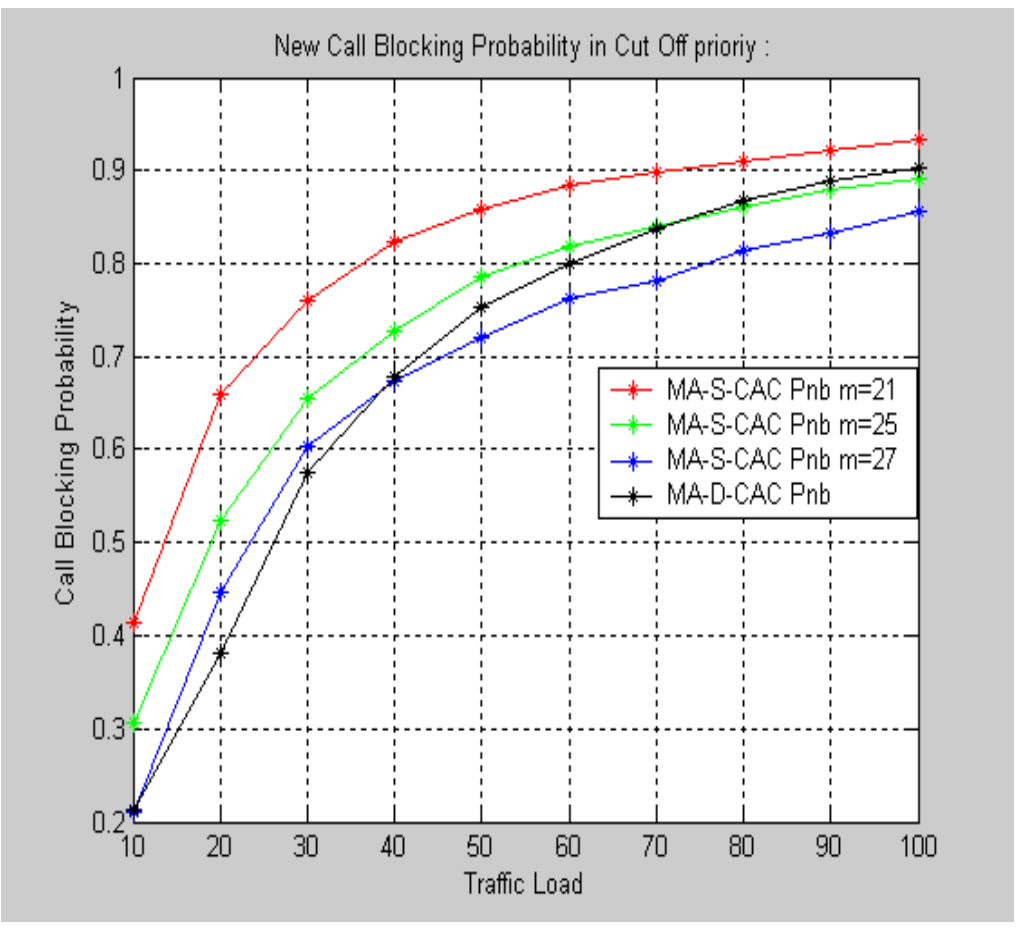

Figure 4 . MA-S-CAC verses MA-D-CAC : $\left(\mathrm{P}_{\mathrm{nb}}\right)$

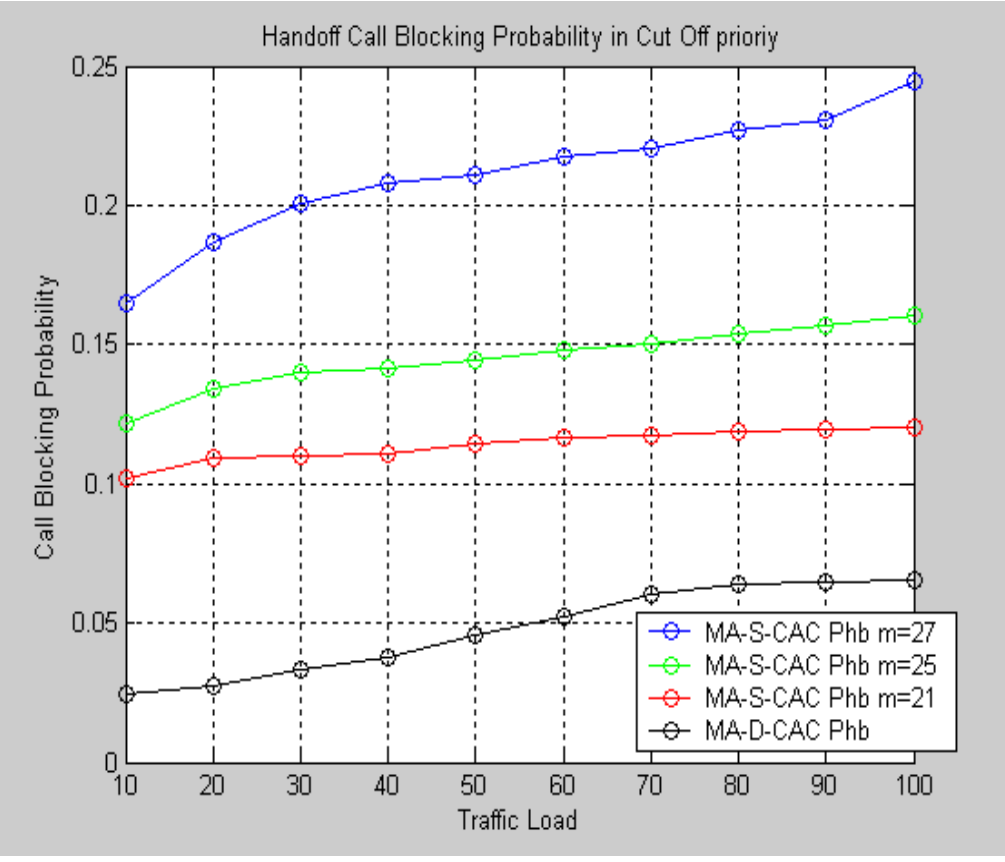

Figure 5. MA-S-CAC verses MA-D-CAC : $\left(\mathrm{P}_{\mathrm{hb}}\right)$ 
The D-CAC though used dynamic cut off threshold which varied according to the number of ongoing calls in the neighboring cell and had less handoff blocking probability as compared to S-CAC (figure 5), but did not consider the impact of mobility pattern of the users, i.e. the blocking probabilities due to the high speed and the low speed calls.

The Dynamic MBCR-CAC was adaptive to the traffic conditions. In this scheme, mobile users were classified according to velocities of the calls, as high-speed users (vehicular users) and low-speed users (pedestrians). This is because the average cell dwell time of a high-speed user is shorter than that of a low-speed user. Based on such a classification, numbers of channels were reserved for handoff calls in target cell. The number of channels reserved was proportionate to the user speed. That is when in a cell high-speed users are more, more number of channels were reserved in neighboring cell to maintain QoS.

Depending on call admission probability two variants of MBCR- CAC schemes were developed. These were Integrated-MBCR and Fractional-MBCR.[13].

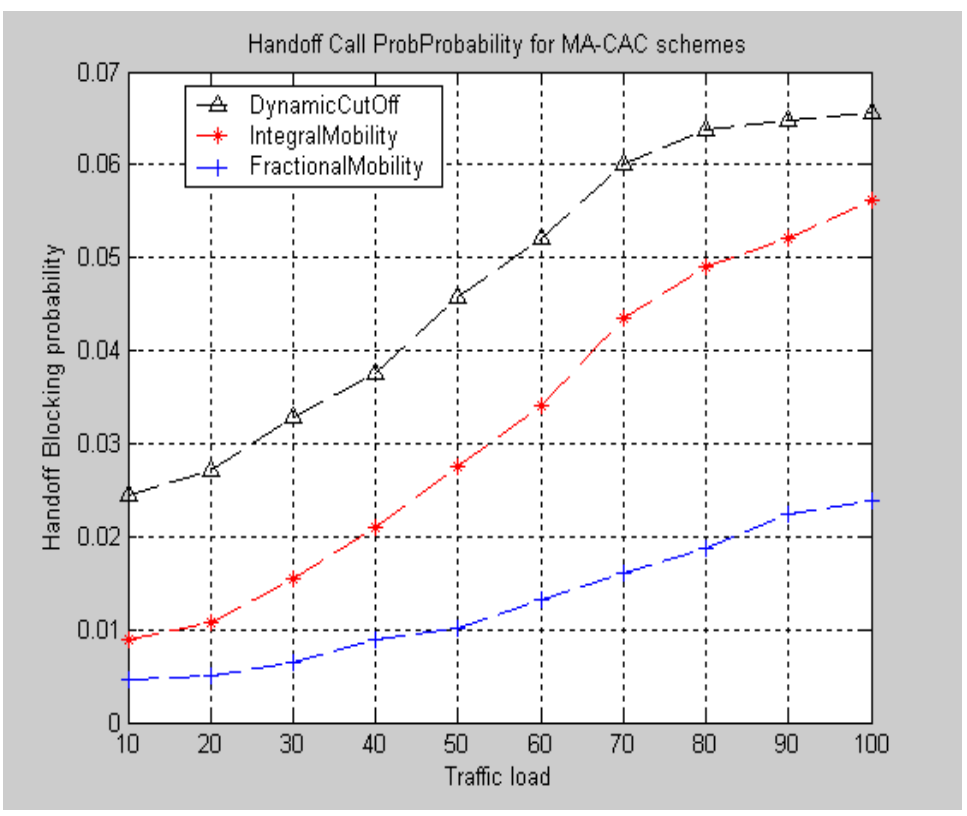

Figure 6. MA-MBCR verses MA-D-CAC : $\left(\mathrm{P}_{\mathrm{hb}}\right)$ 
International Journal of Distributed and Parallel Systems (IJDPS) Vol.3, No.5, September 2012

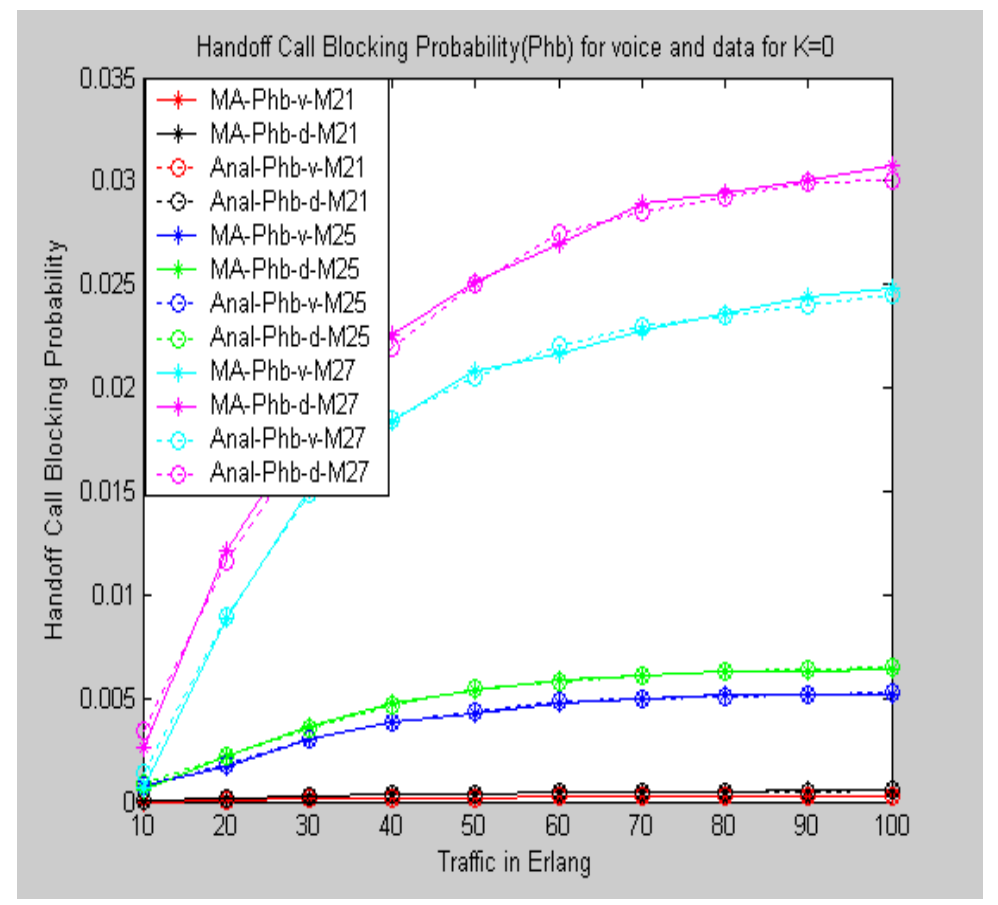

Figure 7. Non-Priority, MA-S-CAC: $\left(\mathrm{P}_{\mathrm{hb}}\right)$

Integral-MBCR being pessimistic as always enough channels were reserved for handoff requests whereas in Fractional-MBCR, depending upon the fraction admission probability $(<$ 0.5 or $>0.5$ ), channel were to be reserved, this gave better performance (figure 6) of handoff blocking probability as compared to other CACs. Thus it was found most suitable for meeting handoff call blocking probability, for highly mobile users moving with the same speed.

To evaluate the performance for multi class traffic, the simulations were also carried out for priority (queue) and non-priority based voice handoff calls. By changing the queue size, it was noticed that the voice handoff blocking probability could be reduced considerably, thus maintaining the voice handoff blocking probability-SLA guarantee. This was shown for S-CAC as well D-CAC strategies as shown figure 7 and figure 8. All these MA-CAC schemes were validated against their analytical models and compared and verified with their non-agent implementations in MATLAB 7.0 for the same simulation parameters. 
International Journal of Distributed and Parallel Systems (IJDPS) Vol.3, No.5, September 2012

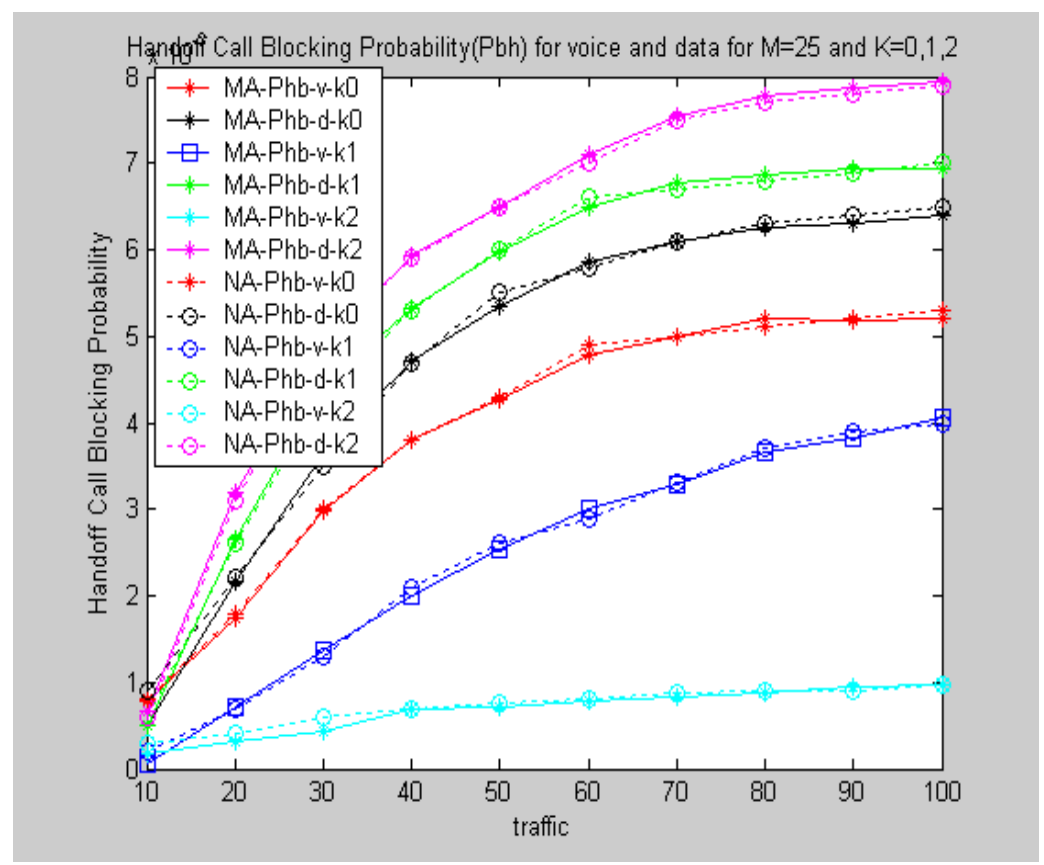

Figure 8. Priority: NA-S-CAC \& MA-S-CAC $(\mathrm{K}=2):\left(\mathrm{P}_{\mathrm{hb}}\right)$

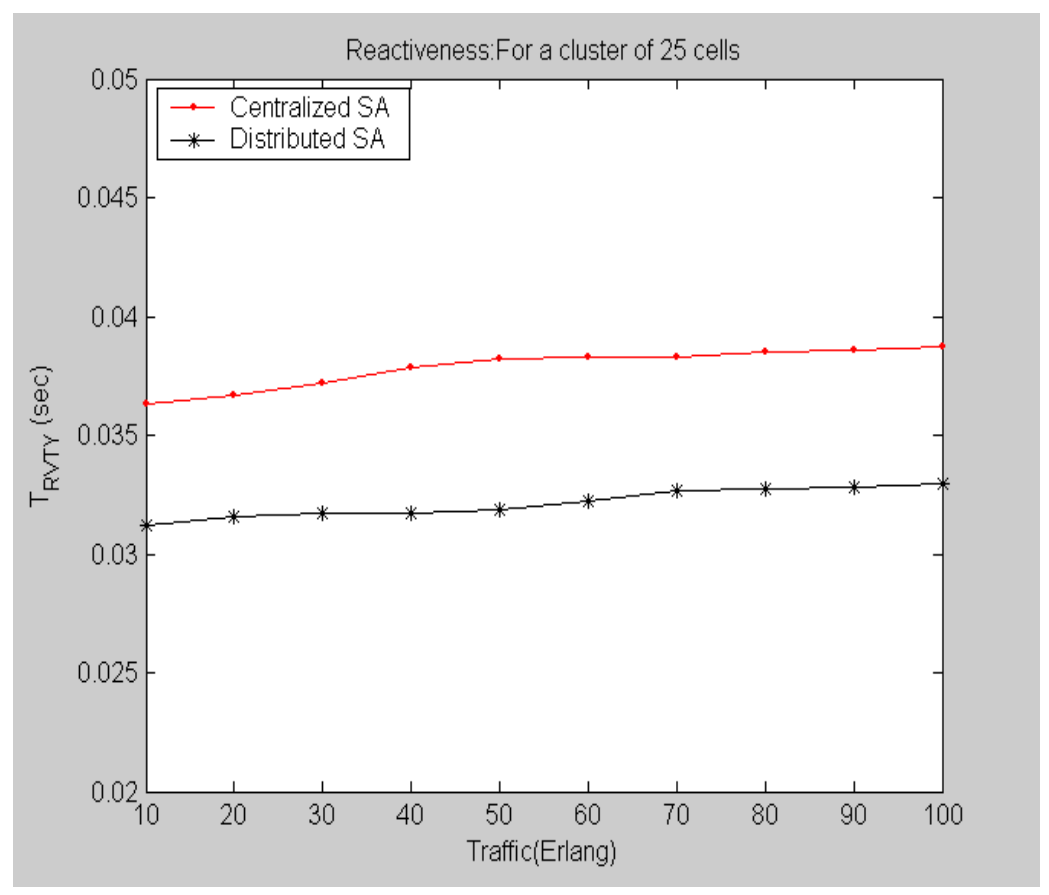

Figure 9. Reactivity of Multi Agent Service Architectures

\subsection{Evaluation of Service Architectures}

According to the degree of distribution of agents, the interaction amongst agents was classified as vertical (Centralised) or horizontal (Distributed), based on which two service architectures were realized. This section presents the discussion on evaluation of these two service architectures. 
Reactivity measured the promptness of the multi agent architectures to react, to the event of call arrival till assignment of the call. It was defined as the sum of the times taken by the agents to interact, make a decision about call admission/rejection by calculating dynamic threshold, and finally allocate a channel to the call, from the time of call arrival. The analysis of the result from figure 9 showed that multi agent based Distributed Service Architecture was more reactive, as compared to Centralized Service Architecture. Even when the load was increased the percentage difference of the reactive ness between the two architectures remained between $13 \%$-15\%.

To measure the effect of increase in traffic on the utilization of resources, average carried load to each cell was measured against the offered load as in figure 10. The results presented that utilization of resources declined with the increase in offered traffic. It was seen that utilization of resources in Distributed Service Architecture was better for higher loads but remained same as that of Centralized Service Architecture for low loads.

To test the scalability of the architectures, the numbers of cells, in clusters of 5 , were increased from 25 to 135 as shown in figure 11 . The results were measured for the reactivity of both the architectures. The reactive time for both the architectures increased almost linearly with increase in the number of cells. The Centralized Service Architecture could not scale in terms of reactivity as time take by it to react with increase in no. of cell agent beyond 115 was very high. This was estimated due to the bottleneck at NPRA whereas Distributed Service Architecture performed relatively well.

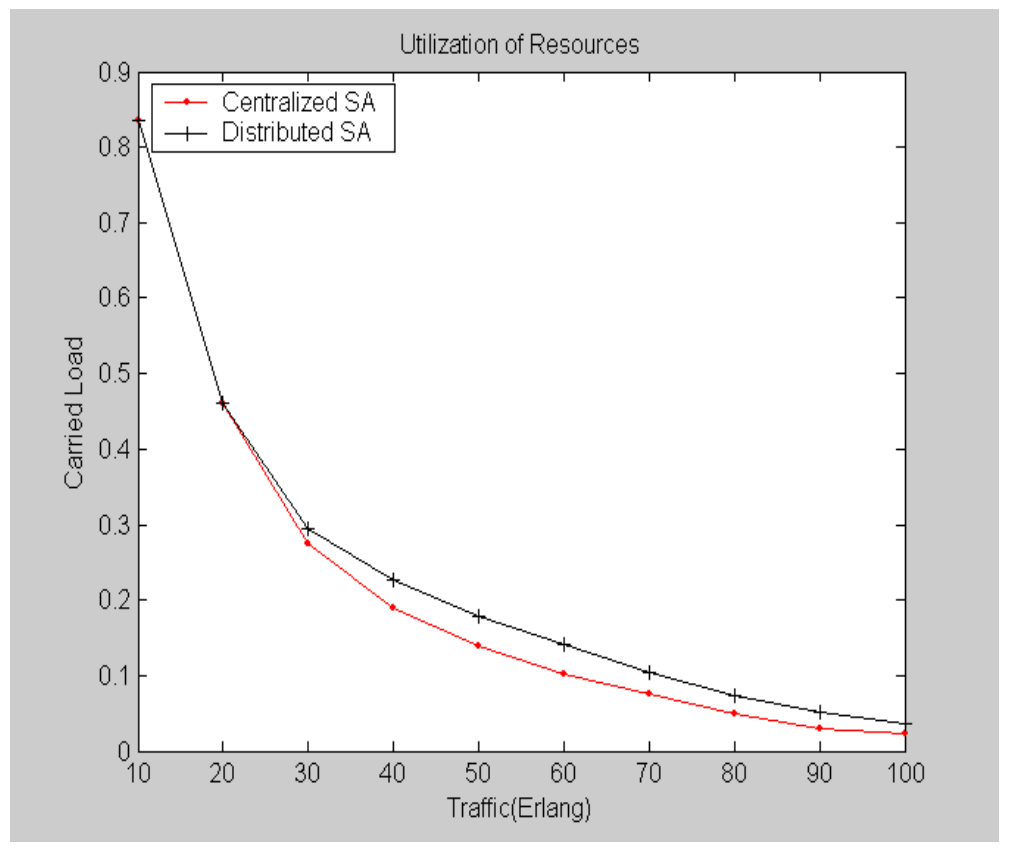

Figure 10. Utilization of Resource: Carried Load verses Offered Load

The message passing between the agents increased the overhead in both the architectures; this was measured by counting the no. of message exchanged per cluster for call admission according the interaction model. Communication overhead in terms of number of messages per call remained approximately 9 (17 in case of congestion control required) messages for Distributed Service Architecture as compared to approximately 12 (22 in case of congestion control required) messages for Centralised Service Architecture. As NPCA cluster was defined as cluster of peer agents, they were present in the same agent container. So the localized nature of the agents resulted in better reactivity for Distributed Service Architecture. 
The sustainability of the two architectures under high traffic intensity was tested. The handoff call blocking probability was chosen as 0.055 toward QoS as in figure 12. It was observed that the Distributed Service Architecture could sustain more traffic load (app. 73 Erlang) as compared to Centralised Service Architecture (app. 69 Erlang) for the same handoff call blocking probability.

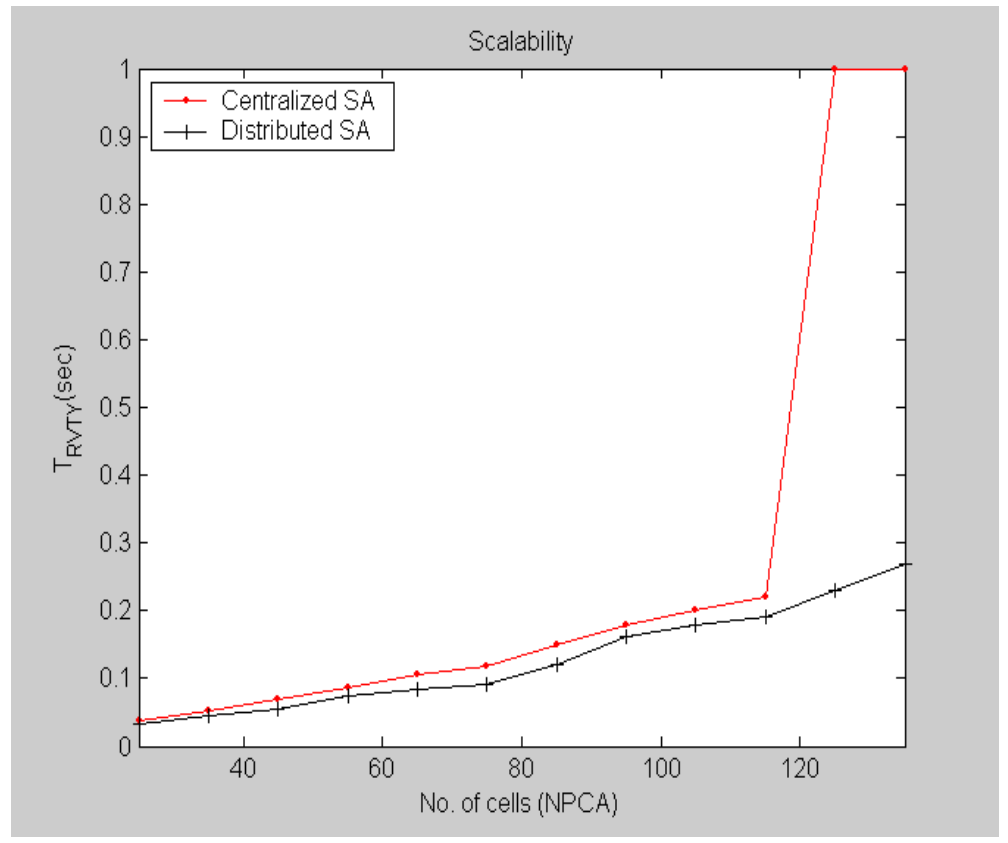

Figure 11. Scalability: Reactivity verses No of Cells

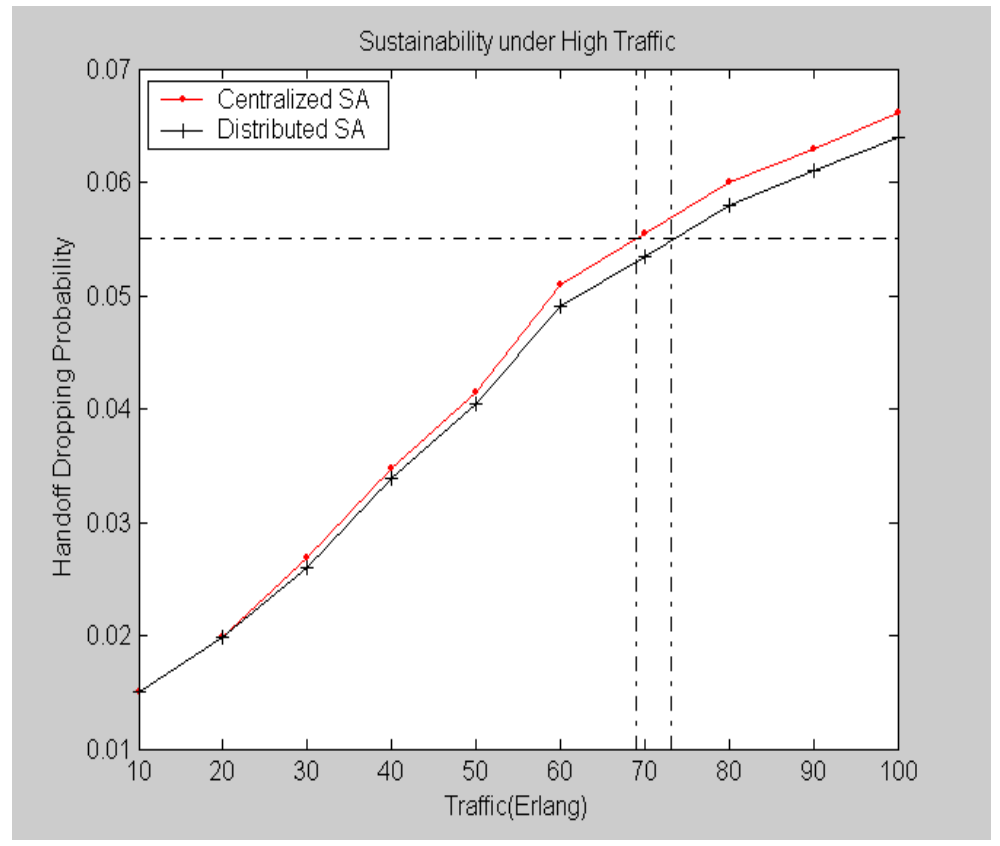

Figure 12. Sustainability: Handoff Blocking Probability verses Traffic 


\subsection{Evaluation of SIA Dynamic Channel Borrowing (MA-DCB)}

This section presents dynamic channel borrowing scheme using SIA based channel borrowing to demonstrate the effectiveness of agents in fair resource distribution through the concepts of utility function [14] and social welfare [15]. The SIAs demonstrate different traits of their attitude towards cluster of agents (society), from being selfish to partially society biased to partially self biased and having balanced attitudes being simulated as SIA- Non-DCB, Partially Society Biased SIA-DCB, Partially Self Biased SIA-DCB and Balanced SIA-DCB respectively.

Fairness of resource distribution was demonstrated by measuring the effect of attitudes on the utility of the system verses utility of agent. The work established the relationship between the utility and call blocking probability as shown in figures 13 and 14. Thus the fairness of resource distribution was defined as a measure of the amount of standard deviation of the call blocking probability of each cell from mean of call blocking probability of the system.

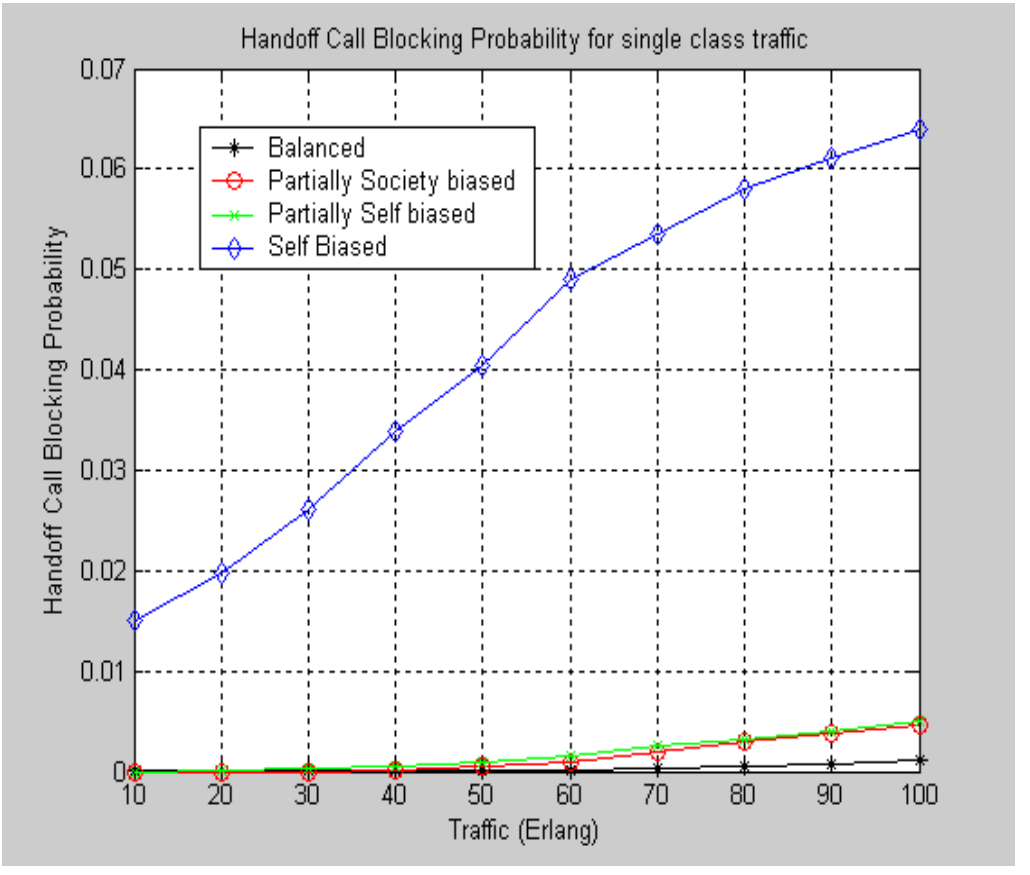

Figure 13. SIA-CB and SIA-Non CB Schemes: $\left(\mathrm{P}_{\mathrm{hb}}\right)$ 


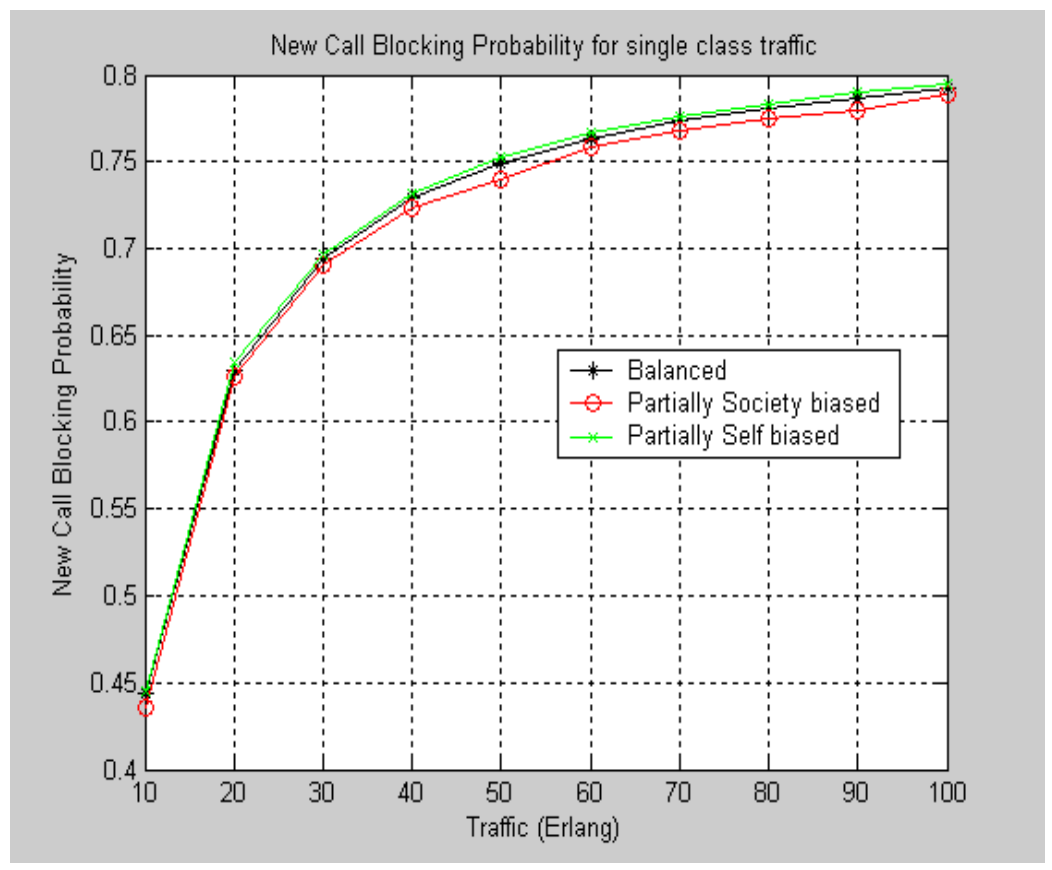

Figure 14. SIA-DCB Schemes: $\mathrm{P}_{\mathrm{hb}}$

It was observed from figure 15 that the SIA-Non DCB behaved like FCA which had the same call blocking probabilities as well as the standard deviation because it did not participate in channel borrowing because of its selfish nature. The 'Balanced' attitude based DCB scheme was fair by $90 \%$, 'Partially Society Biased' by $61 \%$, 'Partially Self Biased' by $56 \%$ as compared to the 'Self-Biased' attitude DCB scheme. This showed that 'Balanced' attitude SIA based MAS not only increase the utility of the system but also are fair in resource distribution to each cell.

\section{CONCLUSION}

The paper presented analysis of the results obtained for MAS based call admission control, channel borrowing strategies as well as service architectures.

The multi agent system designed here can be easily integrated with the Shuffle model, thus making the system extendable and extensible by easily changing/varying the capabilities of the agents according to the type of traffic and required flow control to meet the service level agreement guarantee defined by the network provider. 


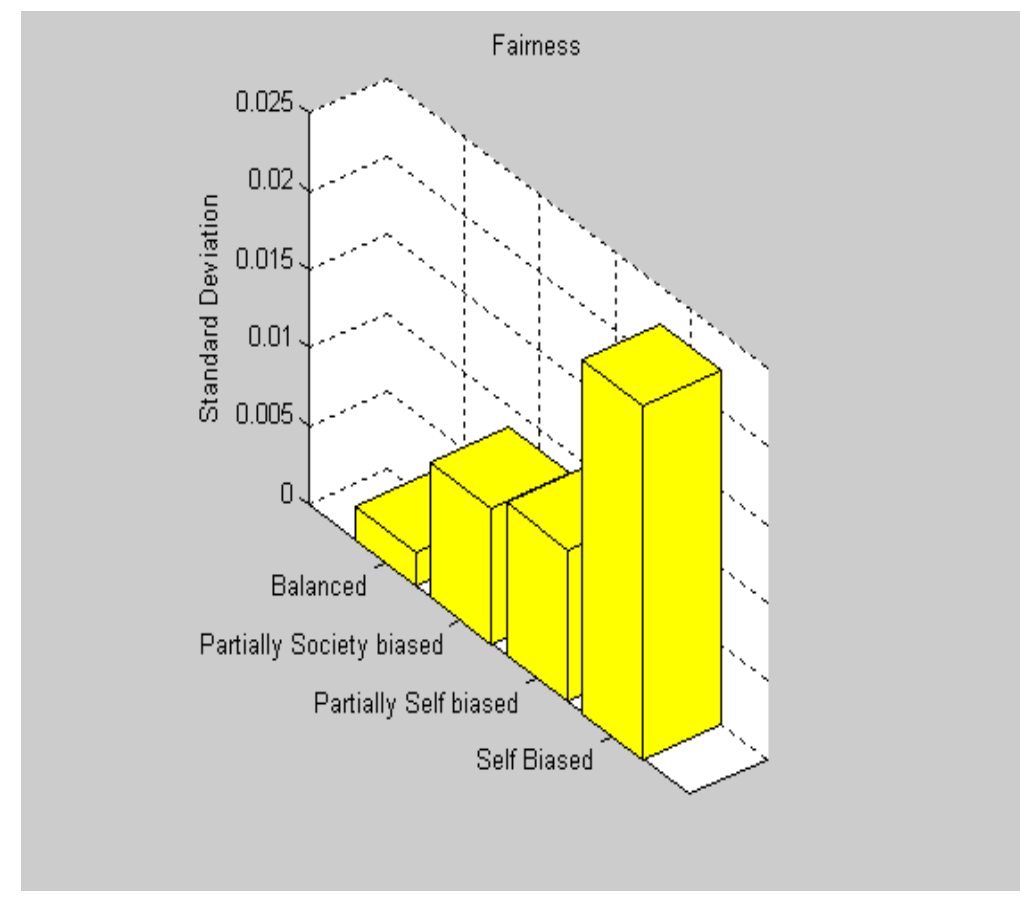

Figure 15. Fairness of Channel Borrowing of SIA-DCB Schemes

The above work establishes that the choice of degree of distribution of agent also plays an important role along with MA-CAC t to maintain the QoS mentioned in SLA. The results presented help in building knowledge for choosing the correct multi agent based CAC or channel borrowing scheme, along with the most suitable service architecture for required QoS and traffic conditions for a cellular network. With the work presented in this paper we hope to assert that multi agent system can be effectively be used in guaranteeing the service level agreement. The challenge is to find right balance of business interests and QoS guarantee and provide timely response, stability in efficient manner. Multi Agent Systems definitely come one step closer to doing just the same.

\section{REFERENCES}

[1] M. Ghaderi and R. Boutaba, "Call admission control in mobile cellular networks: a comprehensive survey," in Proc. of Wireless Communications and Mobile Computing 2006-6993.

[2] Y. Fang and Y. Zhang, "Call Admission Control Schemes and Performance Analysis in Wireless Mobile Network," IEEE Transaction on Vehicular Technology, vol. 51, no. 2, March 2002.

[3] K. Sycara, "Multiagent Systems," Article in AI magazine Vol. 19, (2) Intelligent Agents Summer 1998.

[4] L.Cuthbert, D. Ryan, L.Tokarchuk, J.Bigham, and E. Bodanese, "Using intelligent agents to manage resource in 3G Networks," in Journal of IBTE, 2001, vol. 2 \& 4.

[5] E. Bodanese and L. Cuthbert, "Application of Intelligent Agents in Channel Allocation Strategies for Mobile Networks", In Proc. of the ICC 2000, New Orleans, LA, 2000, pp.181-185.

[6] J. Bigham, L. Cuthbert, A. Hayzelden, and Z. Luo, "Flexible Decentralised Control of Connection Admission,” in Proc. of IMPACT'99, Seattle, WA, ISBN 0-904-18864-7.

[7] A. Bellifemine, R. Hadingham, M. Owen, A. Mamdani, and W. Bouma, "FACTS Project overview : FACTS-FIPA Agent Communication Technologies and Services," ACTS Project AC317, June 19, 1998 at http://www.labs.bt.com/profsoc/facts/. 
International Journal of Distributed and Parallel Systems (IJDPS) Vol.3, No.5, September 2012

[8] J.Bigham, L.Tokarchuk, D. Ryan, L.Cuthbert, J. Lisalina, M. Dinis, and S. Robles, "Agent Based Resource Management for 3G Networks," in Proc. of 3G Mobile Communication Technologies, 26-28 March 2001, Conference Publication No. 477 @ IEE 2001.

[9] N. Giri,, S. Mundle , A. Ray, S. Bodhe: Multi Agent System based Service Architecture for Service Level Agreement for cellular networks ACM 'COMPUTE' 2009 Bangalore.

[10] Java Agent Development Framework, http://www. jade.tilab.com.

[11] C.-J. Chang, T.-T. Su, and Y.-Y. Chiang, "Analysis of a cutoff priority cellular radio system with finite queueing andreneging/dropping," IEEE/ACM Transactions on Networking, vol. 2, no. 2, pp. 166-175, Apr. 1994.

[12] M. Naghshineh and M. Schwartz, "Distributed call admission control in mobile/wireless networks," IEEE Journal on Selected Areas in Communications, vol. 14, no. 4, pp. 711-717, May 1996.

[13] J. Hou, and Y. Fang, "Mobility-based call admission control schemes for wireless," In Proc. of Wireless Communication. Mobile Computing 2001, 1:269-282.

[14] G. Boella, R. Damiano, and L. Lesmo, "Cooperating to the groups utility", in Intelligent Agents VI. Springer Verlag, 1999.

[15] Hogg, L.M. and Jennings, N.R. (1999) Variable Sociability in Agent-Based Decision Making. In: Proc. 6th Int. Workshop on Agent Theories Architectures and Languages (ATAL-99). , Orlando, FL, pp. 305-318. 